\title{
SIMUL@: el uso de mundos virtuales para la adquisición de competencias transversales en la universidad
}

\author{
Francesc Esteve $^{a}$, Vanessa Esteve ${ }^{b}$ y Mercè Gisbert ${ }^{c}$ \\ Rebut: 16/07/2012 Acceptat: 14/09/2012
}

\section{Resumen}

En el presente artículo se pretende compartir la experiencia de una propuesta didáctica llevada a cabo en un mundo virtual, un entorno tecnológico de simulación, para la adquisición y desarrollo de dos determinadas competencias transversales: el trabajo en equipo y la autogestión.

Las competencias, como constructos complejos formadas por conocimientos, habilidades y actitudes, que el sujeto debe ser capaz de poner en acción en contextos determinados. La hipótesis de partida establecida en el proyecto es que los entornos tecnológicos basados en simulaciones son escenarios eficientes para la adquisición y evaluación de estas competencias transversales. Para ello, después de un análisis y conceptualización teórica de dichas competencias se realizó una investigación en la que participaron 72 estudiantes de grado y máster. La actividad didáctica realizada por los sujetos se engloba en 4 fases de 3 semanas de duración: fase preliminar, de desarrollo, de construcción y de evaluación.

En el presente artículo se presentan como resultados las diferentes actividades realizadas por los estudiantes dentro de estos entornos 3D, viendo la tipología, la temporalización y los recursos utilizados en estos metaversos, y se plantean finalmente algunas conclusiones desde el punto de vista del proceso, del profesor y del estudiante.

\footnotetext{
a ARGET: Applied Research Group in Education and Technology (ref. 2009SGR596). Departament de Pedagogia. Universitat Rovira i Virgili - Tarragona

b ARGET: Applied Research Group in Education and Technology (ref. 2009SGR596). Departament de Pedagogia. Universitat Rovira i Virgili - Tarragona

C ARGET: Applied Research Group in Education and Technology (ref. 2009SGR596). Departament de Pedagogia. Universitat Rovira i Virgili - Tarragona
} 
Palabras claves: Educación superior, mundos virtuales, simulación, competencias transversales, trabajo en equipo, autogestión.

\section{SIMUL@: the use of virtual worlds for the acquisition of generic skills in higher education}

\section{Abstract}

The content of this paper is sharing the experience of the organization of a didactic proposal that takes place in a virtual world simulation technology environment for the acquisition and development of two specific generic skills: team-working and self management.

Competencies are complex constructs, consisting of knowledge, skills and attitudes that the subject must be able to put into action in particular contexts. The hypothesis established in the project is that the technological environments are based on model scenarios for the acquisition and efficient evaluation of these transferable skills. To do this, after a theoretical analysis and conceptualization of these skills are carried out research with the participation of 72 students at degree and master. The didactic activity performed by the subjects is included in 4 phases of 3 weeks duration: preliminary phase of development, construction and evaluation.

In this paper results are presented as the various activities undertaken by students within these $3 \mathrm{D}$ environments, seeing the type, timing and resources used in this metaverse, and finally some conclusions arise from the standpoint of process, teacher and student.

Keywords: Higher education, virtual worlds, simulation, key competence, teamwork, self management.

\section{Introducción}

En los últimos años, el aprendizaje y la formación por competencias ha pasado a estar en el centro del debate educativo. Iniciativas como 21st Century Skills (P21, 2011) en el contexto norteamericano, o las denominadas competencias clave para el siglo XXI promovidas por la Comisión Europea (2007) son algunos ejemplos que ponen de manifiesto la importancia de una formación continua, a lo largo de la vida, y centrada en esas competencias transversales que todo ciudadano debe adquirir para hacer frente a las nuevas demandas del entorno.

Estas competencias transversales, tales como la iniciativa, la creatividad, las habilidades comunicativas o, concretamente en las que nos centraremos en este artículo, la autogestión y el trabajo en equipo, frecuentemente son abordadas de manera ligera y superficial en el currículum educativo formal, sin una planificación rigurosa en el proceso formativo y con un peso relativamente inferior a otras 
competencias disciplinares. Además, por la singularidad de este tipo de aprendizajes, estas competencias exigen replantearse nuevas estrategias y nuevos escenarios que permitan al estudiante desarrollarlas plenamente, no sólo demostrando unos conocimientos teóricos, sino poniéndolas en acción, de manera real y ante contextos determinados.

En el presente artículo se pretende compartir la experiencia realizada en un mundo virtual. Una propuesta didáctica realizada en un entorno tecnológico de simulación 3D, para la adquisición y desarrollo de las competencias transversales de trabajo en equipo y autogestión. Esta propuesta didáctica se implementa en el desarrollo de un proyecto de investigación llamado Simul@: Evaluación de un entorno tecnológico de Simulación para el aprendizaje de Competencias Transversales en la Universidad (Ref. EDU2008-01479).

Como veremos a continuación, se trata de una experiencia desarrollada utilizando la plataforma OpenSim, llevada a cabo en alumnos de Magisterio de Educación Física, de Educación Infantil, del Grado de Educación y de Máster de Dirección Estratégica de Empresa.

En base a este estudio, presentaremos indicios de aprendizaje y conclusiones sobre cómo el diseño de actividades dentro del mundo virtual de simulación pueden aportar ideas en la adquisición de competencias transversales y cómo puede ser usado para formar a los estudiantes y profesionales.

\section{Marco Teórico}

El aprendizaje a lo largo de la vida y las competencias transversales

La complejidad del mundo actual y la creciente globalización hacen necesaria una formación que permita a los estudiantes entender cómo funciona este mundo, siempre sin dejar de considerar que la cantidad de información que tendrán que gestionar es cada día mayor y que, para ello, utilizarán en esencia herramientas tecnológicas, que avanzan y cambian a una gran velocidad. Y es que, en palabras de los Ministros de Educación de la OCDE (2005), «el desarrollo sostenible y la cohesión social dependen críticamente de las competencias de toda nuestra población, con competencias que se entiende que cubren el conocimiento, las destrezas, las actitudes y los valores», reflexión que podemos completar aludiendo a otro documento de la Unión Europea, que aborda la palpitante cuestión de las competencias clave. Este documento determina ocho competencias como fundamentales e imprescindibles para cualquier ciudadano, tales como la comunicación en la lengua materna y en lengua extranjera, la competencia digital, la iniciativa o el aprender a aprender, entre otras (Comisión Europea, 2007).

Para abordar esta cuestión, resulta fundamental determinar la naturaleza y las características de las competencias que tendremos que garantizar en los ciudadanos y ciudadanas del siglo XXI. Parece evidente que aquellas competencias básicas que 
todo el mundo tendría que adquirir tienen que ver con (1) entender conceptos complejos, (2) estar alfabetizados digitalmente, (3) adquirir la habilidad de utilizar de modo avanzado las TIC, (4) adquirir las habilidades sociales y comunicativas necesarias para poder desarrollarse social y laboralmente, (5) ser capaz de trabajar en grupo, etc. (Dumont et al., 2010).

A estas competencias se les han Ilamado, de modo genérico, competencias clave. En palabras la propia Comisión Europea (2007), «las competencias clave son aquellas de valor particular que tienen áreas múltiples de utilidad y son necesarias para todos»; y estas deben aportar beneficios en un amplio espectro de contextos $y$, por tanto, han de ser aplicables a diferentes y múltiples áreas del desarrollo vital del ser humano.

Estas competencias, en definitiva, son una combinación de conocimientos, capacidades y actitudes que todas las personas van a precisar para poder desarrollarse personal y profesionalmente, así como para poder convertirse en ciudadanos y ciudadanas activos con un nivel óptimo de inclusión tanto laboral y profesional como social. Si entendemos que éstas son competencias clave, que todo estudiante debe desarrollar a lo largo de su proceso formativo en la universidad, deberemos diseñar estrategias adecuadas que nos permitan, como institución, tener la certeza de que los estudiantes las han adquirido.

\section{La evaluación de las competencias transversales en la universidad}

La evaluación, como parte intrínseca del proceso de enseñanza-aprendizaje, es un procedimiento sistemático de recogida de información y búsqueda de evidencias, que permite la emisión de juicios de valor acerca del logro de unos objetivos determinados, la identificación de lagunas y concepciones erróneas, y la toma de decisiones que permita su certificación (De Miguel, 2000; Cela et al., 2005).

Para tener constancia de la evolución del proceso de aprendizaje del estudiante es necesario hacer uso de una serie de técnicas de evaluación que, a través del uso de determinados instrumentos, permitan recoger información y evidencias. La utilidad de un instrumento en el ámbito de la evaluación radica en su potencialidad para poner de manifiesto aquello que pretende evaluar y su potencialidad real de ser bien utilizado (Salinas, 2002).

Prades (2005), siguiendo los trabajos de Miller et al., (1998) y AQU (2005), afirma que los criterios más relevantes para seleccionar la estrategia y el instrumento de evaluación más adecuado son la coherencia con los objetivos de aprendizaje, la validez y la fiabilidad. Según esta autora, los cuestionarios, los tests o las pruebas de respuesta corta son instrumentos adecuados para comprobar la comprensión, la memorización o aplicación, al implicar habilidades cognitivas, mientras que actividades como las pruebas orales, la elaboración de productos, los portafolios, o los dosieres de aprendizaje, pueden ser muy adecuadas para la evaluación de ejecuciones y comportamientos. 
Las competencias transversales, si las entendemos no sólo como un conjunto de conocimientos, sino tal y como mencionábamos anteriormente, como un constructo complejo formado de conocimientos, habilidades y actitudes en distintas áreas, requerirá la necesidad de establecer procedimientos de evaluación que sean capaces de medir la complejidad de todo este aprendizaje profundo, evitando la simple evaluación de estrategias superficiales de aprendizaje (Simpson, 2003). Para ello, será necesario operativizar la definición de estas competencias, definiendo sus objetivos de aprendizaje (Prades, 2005).

Uno de los instrumentos que pueden ser útiles para este tipo de evaluación son las rúbricas. Una rúbrica es un instrumento en el que se definen unos criterios de valoración y desempeño de un producto, un proyecto, o una tarea en varios niveles progresivos de rendimiento o ejecución (Blanco, 2008; Gil, 2007). Con estos instrumentos, el profesor posee una mayor orientación y apoyo en el análisis, la medición y la correlación de datos durante este tipo de actividades de aprendizaje más complejas, evaluando su logro de manera más eficaz y eficiente (Petropoulo, 2011).

\section{Los entornos virtuales 3D en el aprendizaje y la evaluación de competencias}

Existen diferentes instrumentos para la evaluación de competencias. A menudo, como veíamos en los apartados anteriores, muchos de ellos no permiten la valoración de toda la complejidad que implica una competencia, como integración no sólo de conocimientos sino también de habilidades y actitudes.

El instrumento necesario para este proyecto debe permitir poner en acción estas competencias, y al mismo tiempo, posibilitar la realización de actividades complejas, de múltiples dimensiones de las respectivas rúbricas y de forma simultánea.

Por todo ello, en el siguiente apartado exploramos nuevos espacios tecnológicos que permitan la realización de actividades simuladas más complejas, con las que medir los niveles más elevados de los indicadores de la rúbrica, y trabajar de manera simultánea las distintas alfabetizaciones. Los entornos virtuales 3D, como por ejemplo Second Life u OpenSimulator, son comunidades online que simulan espacios físicos en tres dimensiones, reales o no, y que permiten a los usuarios, a través de sus avatares, interactuar entre sí, y utilizar, crear e intercambiar objetos, y por ende, realizar actividades más complejas, como veremos a continuación.

Atkins (2009) apunta las siguientes características de estos entornos: (1) De inmersión, (2) interactivos, (3) personalizables, (4) accesibles, y (5) programables. Todas estas características permiten la creación de entornos con reglas físicas alternativas, el intercambio síncrono y asíncrono, la alteración de la compresión del tiempo (Allen, 2011), y numerosas potencialidades que pueden resultar muy interesantes para prácticas e investigaciones educativas (Cela et al., 2011). Frente a ello, también se han detectado algunos inconvenientes que pueden dificultar su uso, 
como la poca familiariedad con el entorno y la elevada curva de aprendizaje, el elevado nivel de requisitos técnicos para su funcionamiento o el grado de fiabilidad, confianza y seguridad del sistema (Atkins, 2009). Aspectos, todos ellos, que es necesario tener en cuenta a la hora de diseñar actividades y propuestas educativas.

En estos años, diferentes universidades han empezado a utilizar estas herramientas a nivel educativo. Como apuntan diferentes autores, es necesario explorar su uso más allá de fines publicitarios, clases magistrales y conferencias, potenciando actividades de aprendizaje más centradas en el estudiante (Alrayes, 2011). Allen (2011) expone que estos entornos virtuales 3D pueden utilizarse para las siguientes finalidades: presencia virtual de instituciones, procesos educativos, entrenamiento, planificación de actividades, ensayos, análisis de experiencias, experimentación, pruebas y evaluaciones, y actividades de concienciación. En la presente investigación tratamos de utilizarlas para la realización de una actividad didáctica concreta que nos permita la adquisición de unas determinadas competencias transversales.

\section{Contexto y Diseño de la experiencia}

La experiencia que a continuación se desarrolla se ha llevado a cabo en el marco del proyecto SIMUL@: "Evaluación de un entornos de simulación tecnológicas para el aprendizaje de competencias transversales en la Universidad", realizado por un equipo multidisciplinario de diferentes universidades europeas (Ref. EDU2008-01479).

La hipótesis establecida en el proyecto es que los entornos tecnológicos basados en simulaciones permiten mejorar la adquisición de las competencias transversales de trabajo en equipo y autogestión.

Este proyecto ha sido desarrollado a lo largo de tres cursos académicos (2008-2011) y cuenta con las siguientes fases:

- $\quad$ Parte 1: Diseño

o Definición de las competencias transversales de Trabajo en Equipo y Autogestión, y sus rúbricas de evaluación.

- Análisis de posibles entornos de simulación 3D para su uso en la fase experimental.

- Diseño de la propuesta didáctica a desarrollar con los grupos de estudio.

o Diseño del proceso para evaluar las competencias transversales.

- $\quad$ Parte 2: Experimental

o Proyecto Piloto.

- Fase experimental aplicando la propuesta didáctica en el entorno 3D. 
- Parte 3: Evaluación e informe

o Informe y evaluación de cuentas.

Esta investigación se desarrolló en una Universidad española de tamaño medio (12,000 estudiantes de grado, 900 de master y alrededor de 900 profesores); la cual se caracteriza por tener un campus distribuido en diferentes sedes ofreciendo un amplio abanico de titulaciones tanto oficiales como específicas de la universidad.

\section{Participantes}

En la experiencia participaron 72 estudiantes (72,2\% mujeres y $27,8 \%$ hombres) de una edad media de 24,13 (sd=6,23) correspondientes a titulaciones de Grado de Educación y Magisterio (Educación Infantil: 65,2\%; Educación Primaria: 2,8\%; Educación Física: 15,3; Pedagogía y Educación Social: 4,2\%) y Máster de Dirección de Marketing (12,5\%). Los estudiantes participaron de manera voluntaria y la experiencia tuvo lugar en el contexto de una asignatura correspondiente al currículum ordinario de las titulaciones. Esa asignatura se desarrolló en el segundo cuatrimestre del curso 2010-11.

\section{La propuesta didáctica}

La propuesta didáctica está diseñada para evaluar las competencias transversales de Autogestión y Trabajo en equipo dentro del mundo virtual, independientemente del contexto de la asignatura en que se desarrolle. Para ello, se han categorizado las actividades a desarrollar dentro del mundo virtual, elaboradas a partir de las rúbricas, permitiendo que el profesor las adapte al contexto de su asignatura.

\section{Contexto}

El escenario donde se desarrolla la propuesta didáctica representa casos que simulan el futuro profesional de los estudiantes, orientados a la construcción de un producto determinado. Concretamente tres escenarios: (1) la organización de unas jornadas para un encuentro de escuelas rurales, (2) la organización de unas olimpiadas escolares, y (3) la participación en una feria profesional.

Cada experiencia cuenta con un espacio común en el entorno 3D, llamada isla general, donde realizan las actividades y encuentran información y recursos comunes, y una isla vacía para cada grupo, donde construyen su proyecto. Esta isla vacía tiene tres objetos: (1) un chat, en el que se registra la actividad y las interacciones del grupo, (2) un dispensador de objetos básicos y (3) un dispensador de objetos extra, donde obtienen recursos a cambio de puntos obtenidos en la realización de tareas. 


\section{Entorno virtual 3D}

Los entornos virtuales 3D, como veíamos anteriormente, ofrecen un espacio muy adecuado para el desarrollo de diferentes actividades. Esta experiencia se ha desarrollado en el entorno OpenSimulator, un servidor de aplicaciones 3D de código abierto basado en Second Life que permite construir mundos virtuales.

OpenSim es configurable y permite adaptar las necesidades del proyecto usando módulos. La licencia de OpenSim es BSD. Esta herramienta permite de forma fácil montar un servidor estable, limitando el número de clientes y ser usado en ordenadores más antiguos. El lenguaje que se utiliza para las funcionalidades de los objetos 3D es una variante de C Ilamada LSL. El propio OpenSimulator incluye una herramienta para crear objetos 3D basada en simples figuras geométricas (conocidos como prims o primitivos) y que permite a los residentes la construcción de objetos virtuales y modificar el terreno de la isla. Estas opciones facilitan enormemente nuestra tarea y amplía las posibilidades de interacción con el entorno de los alumnos.

El módulo Sloodle relaciona los mundos virtuales 3D con Moodle. Este módulo ofrece un registro de los avatares dentro de Moodle, que es la plataforma de campus virtual que se utiliza en nuestra universidad. Sloodle soporta OpenSim, permitiendo crear actividades en Moodle que son representadas por objetos 3D en el mundo virtual. Las actividades se registran en Moodle de forma transparente para los participantes. El profesor activa y evalúa las tareas en Moodle y el estudiante puede realizar las actividades dentro del mundo virtual de forma transparente a Moodle, donde se registra el resultado y el seguimiento de la actividad de los usuarios.

\section{Desarrollo de la experiencia}

A continuación se expone cómo se desarrolló la experiencia y cuáles fueron las principales actividades realizadas en estos entornos de simulación 3D, viendo así el potencial y la versatilidad de estos entornos.

\section{Temporalización}

La propuesta se engloba en 4 fases de 3 semanas de duración, formadas por diferentes actividades, dividas a su vez en una serie de tareas:

\section{Fase preliminar (presencial, 3 horas):}

- $\quad$ Actividad 0: "Introducción". Instalación, explicación de la herramienta y de las normas básicas.

- $\quad$ Actividad 1: "Personalización del avatar".

- $\quad$ Actividad 2: "Familiarización con el entorno". 
- $\quad$ Actividad 3: "Conocernos mejor". Actividad introductoria, en la que rellenan una serie de cuestionarios (patrones de aprendizaje, competencias transversales y competencia digital) y se reorganiza los grupos de trabajo en función de los resultados.

\section{Fase de desarrollo}

En esta fase los estudiantes deberán realizar diferentes tareas para obtener puntos que podrán canjear por objetos extra en la construcción de su proyecto. Estas actividades están relacionadas con las rúbricas en el contexto de su asignatura y orientadas a la construcción.

Hay una tarea común en todos los grupos que es Actividad 4: Mi equipo: responsables y objetivos por ámbito, en la que los estudiantes deben determinar los responsables de cada ámbito del proyecto y asignar los objetivos a alcanzar.

El resto de actividades son individuales o grupales y tendrán un contador que se incrementa con la nota de las tareas hasta la fecha de inicio de construcción de proyecto. La tipología de actividades según las rúbricas es: (1) Organización personal,

(2) Organización espacial, (3) Organización documental, (4) Elaborar listados, (5) Elaborar presupuestos, (6) Elaborar calendario, (7) Programar/anticipar, (8) Explicar/argumentar.

Asimismo, los objetos Sloodle que se han usado para realizar las tareas són: (1) Quizchair, para realizar cuestionarios, (2) Metagloss, glosario de apoyo, (3) Blog, los estudiantes llevaron un diario de la experiencia, (4) Webintercom, es el chat que usaban los estudiantes para comunicarse entre ellos en todas las fases. Era un requisito obligatorio, porque a partir de él, el profesor podía evaluar la toma de decisiones en el grupo y servía de registro de toda la interacción del mundo, (5) Presentations, se utiliza de panel para exponer información a los estudiantes sobre el proyecto en general y como tablón de anuncios, (6) Primdrop: Entrega de tareas.

\section{Fase de construcción}

En esta fase, los estudiantes empiezan a organizar el espacio y construir en su isla, para ello podrán crear objetos, utilizar y modificar objetos de los dispensarios y gestionar los puntos obtenidos en la fase de desarrollo.

El resultado de esta fase es presentar su isla como producto para ser evaluado delante de un comité. En la Figura 1 se puede ver un ejemplo de los estudiantes trabajando en sus proyectos. 


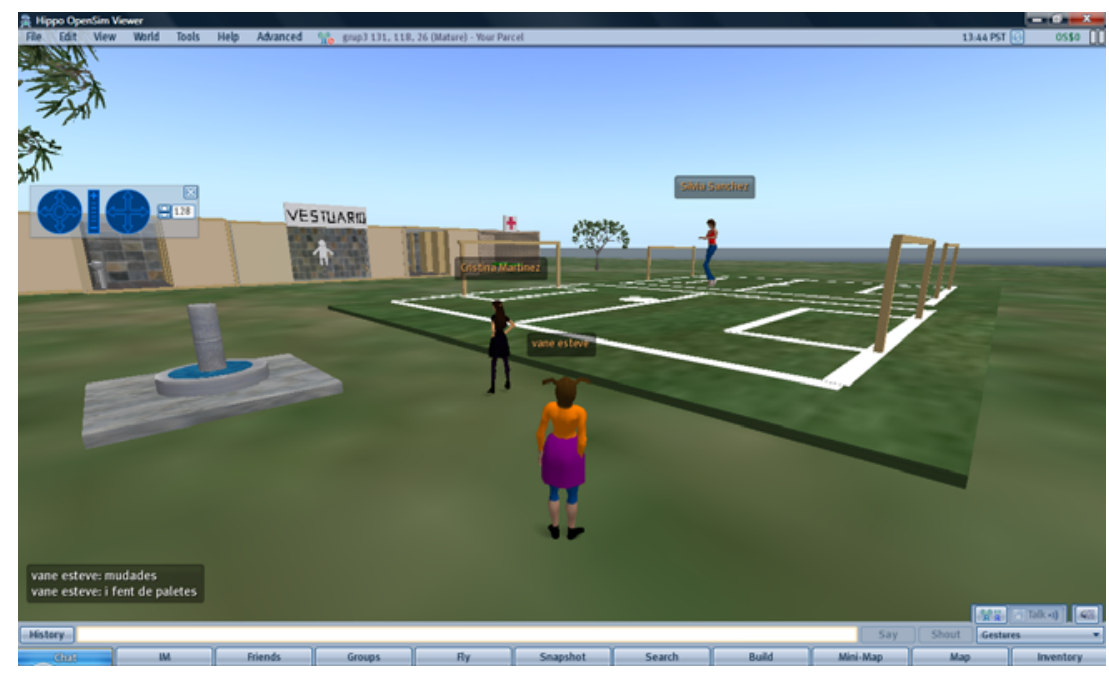

Figura 1: Estudiantes trabajando en sus proyectos (Fuente: elaboración propia)

\section{Fase de evaluación}

En esta fase los estudiantes presentan su isla ante el Comité Evaluador y el resto de equipos, justificando el proyecto con respecto al contexto de la asignatura y las actividades realizadas en la fase de desarrollo.

A continuación se realiza una votación donde el resto de equipos valoran su trabajo y realizan unos cuestionarios a partir de las rúbricas donde se evalúan las competencias de Autogestión y trabajo en equipo. Hay diferentes cuestionarios: (1) Autopercepción del efecto del simulador en cuanto a las competencias, (2) Co-evaluación, donde los estudiantes se evalúan entre los miembros del equipo, y (3) Heteroevaluación, en el que los profesores y supervisores evalúan a los grupos.

Durante toda la experiencia, los grupos han contado con consultores que estaban disponibles en el mundo y ayuda técnica por correo electrónico y los foros de la asignatura en Moodle. El rol de los consultores consiste en resolver dudas o necesidades que el grupo podía tener, ya que no tuvieron una formación explícita en el entorno.

\section{Desarrollo de las actividades}

La propuesta didáctica, como veíamos anteriormente, está orientada a la construcción de un determinado producto: organización de unas jornadas para un encuentro de escuelas rurales, organización de unas olimpiadas escolares o participación en una feria profesional. 
Sin embargo, para exponer la experiencia realizada, se ha organizado la información desde tres puntos de vista: el proceso, el profesor y el estudiante.

\section{El proceso}

A partir de las rúbricas se analizaron aquellos aspectos importantes a trabajar en la propuesta didáctica y que el mundo virtual puede dar respuesta. Por tanto, se diseñan juntamente con el profesor unas actividades a partir de la categorización de la rúbrica. El profesor activa en Moodle las actividades y mediante Sloodle se representan en el entorno virtual 3D (OpenSim).

Esta experiencia se ha realizado en diferentes contextos, a continuación presentamos las actividades y las fichas de los recursos utilizados:

\section{Olimpiada Escolar}

En la presente actividad (Tabla 1 ) se trata de organizar una olimpiada escolar de 5 días de duración, con un programa deportivo y de actividades sociales que recoja todas las especialidades deportivas que oferta el centro. El proyecto se tendrá que presentar y defender ante un comité olímpico.

\begin{tabular}{|c|c|c|}
\hline \multicolumn{3}{|c|}{ OLIMPIADA ESCOLAR } \\
\hline $\begin{array}{l}\text { DESCRIPCIÓN Y } \\
\text { CONTEXTOO }\end{array}$ & \multicolumn{2}{|c|}{$\begin{array}{l}\text { Se trata de un centro escolar de educación primaria, secundaria y } \\
\text { bachillerato, con tres grupos de } 25 \text { alumnos por cada curso. } \\
\text { Tiene un programa de deporte extraescolar con el que diferentes } \\
\text { equipos participan en competición escolar y federada. Actualmente } \\
\text { dispone de los siguientes equipos y equipamiento deportivo. }\end{array}$} \\
\hline $\begin{array}{l}\text { ÁMBITOS DEL } \\
\text { PROYECTO }\end{array}$ & \multicolumn{2}{|c|}{$\begin{array}{l}\text { - Seguridad, control y atención médico-sanitaria. } \\
\text { - Difusión, publicidad y gestión de recursos económicos. } \\
\text { - Organización de la competición deportiva. }\end{array}$} \\
\hline RECURSOS & $\begin{array}{l}\text { BÁSICOS: } \\
\text { - Pista de Vóley. Equipos juvenil } \\
\text { (masc. y fem.) y junior masc. } \\
\text { - Pista Futbol-sala: Equipos juvenil } \\
\text { y junior masc. } \\
\text { - Pista de tenis. } \\
\text { - Gimnasio cubierto polivalente de } \\
30 \times 20 \text { dotado de espalderas, } \\
\text { espejos, equipo de música y un } \\
\text { tatami móvil. } \\
\text { - Centro médico. } \\
\text { - Balón de fútbol, vóley y pelotas } \\
\text { de tenis. } \\
\text { - Vestuario }\end{array}$ & $\begin{array}{l}\text { EXTRA: } \\
\text { - Bar: } 20 \\
\text { - Grada: } 10 \\
\text { - Megafonía: } 10 \\
\text { - Tienda de suvenires: } 10 \\
\text { - Pódium: } 10 \\
\text { - Publicidad: } 10 \\
\text { - Trofeos: } 30(15+10+5)\end{array}$ \\
\hline
\end{tabular}

Tabla 1: Propuesta didáctica "Olimpiada Escolar" (Fuente: Elaboración propia) 


\section{Jornada de Escuelas Rurales}

En la siguiente actividad didáctica (Tabla 2) se trata de organizar una jornada de encuentro de escuelas rurales, formada por 3 escuelas, 2 cíclicas y 1 unitaria. Las escuelas cíclicas disponen de 45 alumnos de infantil (15 por nivel) y 60 de primaria (20 por cada ciclo) cada escuela respectivamente. La escuela unitaria dispone de 8 alumnos de infantil y 6 de primaria.

\begin{tabular}{|c|c|c|}
\hline \multicolumn{3}{|c|}{ JORNADA DE ESCUELAS RURALES } \\
\hline $\begin{array}{l}\text { DESCRIPCIÓN Y } \\
\text { CONTEXTO }\end{array}$ & \multicolumn{2}{|c|}{$\begin{array}{l}\text { Decidir el tipo de escuela que se necesita y el centro de interés de la } \\
\text { jornada. } \\
\text { Escoger los diferentes espacios en función del alumnado, las actividades } \\
\text { y necesidades }\end{array}$} \\
\hline $\begin{array}{l}\text { ÁMBITOS DEL } \\
\text { PROYECTO }\end{array}$ & \multicolumn{2}{|l|}{$\begin{array}{l}\text { - Seguridad y control. } \\
\text { - Responsabilidades y atención. } \\
\text { - Difusión y publicidad. } \\
\text { - Gestión de recursos económicos. } \\
\text { - Organización de la jornada de la ZER. }\end{array}$} \\
\hline RECURSOS & $\begin{array}{l}\text { BÁSICOS: } \\
\text { - Pista de Vóley. Equipos juvenil } \\
\text { (masc. y fem.) y junior masc. } \\
\text { - Pista Futbol-sala: Equipos juvenil } \\
\text { y junior masc. } \\
\text { - Pista de tenis. } \\
\text { - Gimnasio cubierto polivalente de } \\
30 \times 20 \text { dotado de espalderas, } \\
\text { espejos, equipo de música y un } \\
\text { tatami móvil. } \\
\text { - Centro médico. } \\
\text { - Balones de fútbol, vóley y pelotas } \\
\text { de tenis. } \\
\text { - Vestuarios }\end{array}$ & $\begin{array}{l}\text { EXTRA: } \\
\text { - cuerdas: } 10 \\
\text { - Tiza: } 10 \\
\text { - Megafonía: } 10 \\
\text { - Equipo de música: } 10 \\
\text { - Objetos de recuerdo: } 10 \\
\text { - Gradas: } 10 \\
\text { - trofeos: } 10 \\
\text { - Zona mural y pinturas:10 } \\
\text { - Pañuelos: } 10 \\
\text { - Zancos: } 10 \\
\text { - Hinchables: } 20 \\
\text { - Payasos: } 20 \\
\text { - Nevera: } 20\end{array}$ \\
\hline
\end{tabular}

Tabla 2: Propuesta didáctica "Jornada de Escuelas Rurales" (Fuente: Elaboración propia)

\section{Ferias profesionales}

En esta última actividad didáctica (Tabla 3) se busca participar en una feria de 4 días de duración, para presentar y ofrecer un nuevo producto y/o servicio. 


\begin{tabular}{|c|c|c|}
\hline & \multicolumn{2}{|l|}{ FERIAS PROFESIONALES } \\
\hline $\begin{array}{l}\text { DESCRIPCIÓN Y } \\
\text { CONTEXTO }\end{array}$ & \multicolumn{2}{|c|}{$\begin{array}{l}\text { Las ferias son instrumentos de apoyo para el logro de objetivos en el } \\
\text { campo de promoción y venta y, como tales, deben situarse en el } \\
\text { contexto de un plan de marketing que establezca objetivos claros y } \\
\text { concretos. } \\
\text { La participación en una feria nos ofrece la oportunidad para exponer y } \\
\text { promover la oferta de productos y servicios de una empresa. Deben } \\
\text { organizarse y planificarse correctamente para obtener los beneficios que } \\
\text { nos brindan. } \\
\text { Este proyecto trata de la presentación de un nuevo producto en una feria } \\
\text { del sector. Se tiene que preparar la participación en una feria profesional, } \\
\text { sin embargo durante el fin de semana está abierto para el público en } \\
\text { general. Por ello, será necesario preparar un programa con diferentes } \\
\text { actividades para ambos públicos }\end{array}$} \\
\hline $\begin{array}{l}\text { ÁMBITOS DEL } \\
\text { PROYECTO }\end{array}$ & \multicolumn{2}{|l|}{$\begin{array}{l}\text { - Creativo } \\
\text { - Financiero } \\
\text { - Logístico e infraestructura }\end{array}$} \\
\hline RECURSOS & $\begin{array}{l}\text { BÁSICOS: } \\
\text { - Mesa estándar } \\
\text { - Silla } \\
\text { - Banco } \\
\text { - Estantería } \\
\text { - Mostrador } \\
\text { - Moqueta } \\
\text { - Ordenador y monitor } \\
\text { - Equipo audiovisual } \\
\text { - Decoración (floral, ornamental) } \\
\text { - Obsequio } \\
\text { - Armario } \\
\text { - Perchero }\end{array}$ & $\begin{array}{l}\text { EXTRA: } \\
\text { - Cafetera: } 2 \\
\text { - Nevera: } 4 \\
\text { - Puf: } 2 \\
\text {-Expositores: (bandera, display, } \\
\text { póster): } 2+2+2 \\
\text { - Barra: } 4 \\
\text { - Mesa redonda: } 2 \\
\text { - Taburete: } 2 \\
\text { - Paneles: } 2 \\
\text { - Catering: } 4 \\
\text { - Iluminación extra: } 2 \\
\text { - Urna: } 4 \\
\text { - Impresora: } 2 \\
\text { - Personal de atención al público: } 4\end{array}$ \\
\hline
\end{tabular}

Tabla 3: Propuesta didáctica "Ferias Profesionales" (Fuente: Elaboración propia)

\section{El profesor}

El papel del profesor en el proyecto es fundamental en las diferentes fases de la experiencia. En un inicio, el profesor define la finalidad y los objetivos del proyecto y describe el contexto: el escenario del proyecto. El profesor diseña aquellos aspectos importantes a trabajar en la experiencia y toda la información relacionada con el proyecto que recibe el estudiante.

Posteriormente, el profesor define la propuesta de actividades según el formato (si son tareas de Moodle, objetos Sloodle) y según la tipología (actividades de organización, de elaboración, de anticipación, de explicación o justificación). Una vez realizadas las tareas, el profesor debe evaluarlas y dar los puntos para obtener recursos extra. También debe definir qué objetos son básicos y qué objetos son extra, 
acompañados de la puntuación individual. En la evaluación, el profesor forma parte del comité evaluador y debe evaluar las competencias a modo de heteroevaluación.

\section{El estudiante}

Finalmente se presenta la secuencia que el estudiante tiene que seguir para desarrollar el proyecto con éxito.

- Sesión inicial: Instalación del visor, configuración del grid, personalización de su avatar, familiarización con el entorno, realización de los cuestionarios previos para la formación de grupos

- Actividades: Realización de las tareas y acumulación de puntos

- Construcción: Gestión de los recursos, obtención de recursos nuevos (por creación, por dispensario) y uso de los recursos (aplicar propiedades de textura, subir imágenes)

- Evaluación: Presentación del proyecto, evaluación (co-evaluación: evaluación al resto de integrantes del grupo y autopercepción)

\section{Conclusiones de la experiencia}

Este artículo trata de describir un proceso caracterizado por uno de los elementos que constituyen la base de su contenido: aprender haciendo. Es necesario explorar nuevas estrategias formativas, que permitan poner en acción los aprendizajes para poder asegurar el desarrollo y la adquisición de competencias.

Como hemos visto tras el análisis documental, los entornos virtuales 3D son espacios modulables, programables, interactivos, y de inmersión, que permiten albergar la realización de actividades complejas, registrando con datos empíricos toda la actividad realizada en ellos. Éstos, además generar un contexto simulado en el que los estudiantes pudieran desarrollar sus actividades, han permitido situar al estudiante en el proceso de aprendizaje, siendo, de todas maneras, el profesor quien diseña y guía la actividad. Asimismo, el entorno tecnológico ha facilitado el proceso de evaluación por medio de un sistema de registro de toda la actividad realizada. Este proceso debe ser planificado y previsto a fin de recopilar la información más relevante para regular el proceso y tomar buenas decisiones con respecto a resultados de la enseñanza.

En cuanto a la tecnología, constituye un verdadero reto observar otras experiencias en mundos virtuales que utilizan una secuencia didáctica igual o similar a la que hemos propuesto. En este sentido, herramientas como por ejemplo Unity 3D ofrecen una buena oportunidad para seguir esta idea. Unity 3D es una herramienta de desarrollo de videojuegos basada en el cliente que provee de un editor visual muy útil y completo donde mediante unos pocos clics podremos importar nuestros 
modelos 3D, texturas, sonidos, etc. para después ir trabajando con ellos. Además, permite el desarrollo para otras plataformas, e incluso trasladar nuestro desarrollo a dispositivos móviles, algo que creemos puede ser interesante analizar para próximas investigaciones. Sin embargo, el uso de OpenSim nos ha permitido tener una adecuada compatibilidad con la plataforma Moodle, algo que sin duda nos parece muy relevante en este contexto.

\section{Referencias}

AQU (2005). Estàndards d'acreditació de les titulacions. Agència per a la Qualitat del Sistema Universitari de Catalunya (AQU).

Allen, P.D. y Demchak, C.C. (2011). Applied Virtual Environments: Applications of Virtual Environments to Government, Military and Business Organizations. Journal of Virtual Worlds Research, 4(1).

Alrayes, A. y Sutcliffe, A. (2011). Students Attitudes in a Virtual Environment (SecondLife). Journal of Virtual Worlds Research, 4(1).

Atkins, C. (2009). Virtual Experience: Observations on Second Life. En Purvis, M. y Savarimuthu, B. (Eds.), Lecture Notes in Computer Science: Vol. 5322. ComputerMediated social networking. Springer Berlin / Heidelberg.

Blanco, A. (2008). Las rúbricas: un instrumento útil para la evaluación de competencias. En Blanco, A., Morales, P. Y Torre, JC "La enseñanza universitaria centrada en el aprendizaje: estrategias útiles para el profesorado". Barcelona: Octaedro-ICE UB.

Cela, J., Esteve, V., Marqués, L., Gisbert, M., Arias, I., Vaca, B.E. y Samaniego, G.N. (2011). SIMUL@: 3D Spaces to Learn Generic Skills. A Pilot Study With Education Students. En 6th international conference on e-learning. Canada: University Of British Columbia.

Cela, J.M., Fandos, M., Gisbert, M. y González, P. (2005). Adaptación de titulaciones al EEES: un ejercicio metodológico. Revista electrónica interuniversitaria de formación del profesorado, 21(8-6), 17-22.

Comisión Europea (2007). Competencias clave para el aprendizaje permanente. Un marco de referencia europeo.

De Miguel, M. (2000). La evaluación de programas sociales: fundamentos y enfoques teóricos. Revista de investigación educativa, 18(2), 289-317.

Dumont, H., Istance, D. y Benavides, F. (2010). The nature of learning. Using research to inspire practice. Centre for Educational Research and Innovation (CERI). OECD.

Gil Flores, J. (2007). La evaluación de competencias laborales. Educación XXI: Revista de la Facultad de Educación (10), 83-106. 
Miller, A.H., Imrie, B.W. y Cox, K. (1998). Student assessment in higher education: A handbook for assessing performance. Routledge.

OECD (2005). The definition and selection of key competencies (DeSeCo). Executive summary.

P21 (2011). P21 common core toolkit. A guide to aligning the common core state standards with the framework for 21st century skills. The Partnership for 21st Century Skills.

Petropoulou, O., Vassilikopoulou, M. y Retalis, S. (2011). Enriched assessment rubrics: a new medium for enabling teachers to easily assess student's performance when participating in complex interactive learning scenarios. Operational Research, 11171186.

Prades, A. . (2005). Les competències transverals i la formació universitària. Thesis, Universitat de Barcelona.

Salinas, D. (2002). ¡Mañana examen! La evaluación: Entre la teoría y la realidad. Grao.

Simpson, R.D. (2003). A Search for the Complete Education: Balancing the Needs for Survival and Fulfillment. Innovative Higher Education, 28(2), 91-105.

Dirección de contacto: Laboratori d'Aplicacions de la Tecnologia a l'Educació. Facultat de Ciències de l'Educació i Psicologia. Departament de Pedagogia. Universitat Rovira i Virgili. Carretera de Valls, s/n 43007 Tarragona (España) 43007 Tlfn: + 34977558466

Francesc M. Esteve Mon. Es licenciado en Psicopedagogía y diplomado en Magisterio por la Universitat Jaume I (UJI), y máster en Tecnología Educativa por la Universitat Rovira i Virgili (URV). Actualmente es personal investigador en formación de la URV, donde está desarrollando su tesis doctoral. Es evaluador en diferentes programas de la Agencia Nacional de Evaluación de la Calidad y Acreditación (ANECA), y colabora asimismo con la Cátedra UNESCO de Gestión y Política Universitaria de la Universidad Politécnica de Madrid, y con el Centre d'Educació i Noves Tecnologies (CENT) de la UJI.

E-mail: francescmarc.esteve@urv.cat

Vanessa Esteve González. Es ingeniera técnica en informática de gestión y ha realizado el máster en Tecnología Educativa: e-learning y gestión del conocimiento, por la Universidad Rovira i Virgili (URV). Es personal técnico de soporte a la investigación del grupo ARGET- Applied Research Group in Education and Technology (ref. 2009SGR596) del Departamento de Pedagogía de la URV.

E-mail: vanessa.esteve@urv.cat 
UT. Revista de Ciències de l'Educació Desembre 2012. Pag. 7-23

Mercè Gisbert Cervera. Es doctora en Filosofía y Ciencias de la Educación por la Universitat de Barcelona. Actualmente es profesora del Área de Didáctica y Organización Escolar del Departamento de Pedagogía de la Universitat Rovira i Virgili (URV). Ha desempeñado diferentes cargos de gestión, entre los que destacar, vicerrectora de Política Docente y EEES, vicedecana de la Facultad de Ciencias de la Educación y Psicología y directora del Instituto de Ciencias de la Educación. Es coordinadora del doctorado de Tecnología Educativa de la URV, del grupo de investigación Applied Research Group in Education and Technology (ARGET) y del Laboratori d'Aplicacions de la Tecnologia a l'Educació (L@TE).

E-mail: merce.gisbert@urv.cat 